\title{
Aggregation of Systematic Operational Capability of Armaments
}

\author{
Xinhua He,Yinan Ma, Qiong Wang \\ Department of Information Engineering \\ Academy of Armored Forces Engineering \\ Beijing 100072, china \\ e-mail:hxh717495@tom.com
}

\begin{abstract}
Aggregation of systematic operational capability of armaments is significant for armaments systems' developing, constructing and using. As holistic avalanche and operational capability's nonlinear character of armaments systems, the feedback mechanism among capability indexes is researched and an aggregate method of systematic operational capability based on feedback mechanism is proposed on this paper, what's more, the method is proved effective by certain example of reconnaissance intelligence armaments systems' operational capability aggregation.
\end{abstract}

Keywords-armaments systems; nonlinear; feedback mechanism; operational capability aggregation

\section{INTRODUCTION}

Information warfare emphasizes the confrontation between the system and the system. The system ${ }^{[1]}$,also called "system of systems", it is possible to obtain a collection of further emerged nature associated or links independent systems. Weaponry system ${ }^{[2]}$ in certain state guidance, operational command and security conditions for the completion of certain operational tasks by function with each other, the higher levels of system interaction of various types of system interaction of various types of weapons and equipment systems. The weaponry system operational capability polymerization research work, the future development of weaponry and equipment system, planning development and use has a pivotal role, is one of the hot spots of the field of military research.

Most of the combat capability platform-system centric operational capability analysis, ignore the multi-network interaction between the various components within the system.

Linear superposition of the combat capability of multiple platforms-system can not achieve the overall emergence of the combat capability polymerization can not reflect the nonlinear characteristics of the elements of the combat capability.

Author first polymerization characteristics of the operational capability of the weapon equipment system, the concept of feedback on this basis will be introduced into the combat capability polymerization feedback mechanism between capacity indicators proposed a feedback mechanism-based weaponry the combat capability polymerization method, and the example analysis and validation

\section{COMBAT CAPABILITY AGGREGATION}

\section{A. Capability polymerization Characteristsces}

Weaponry system operational capability ${ }^{[3]}$ weaponry system is required to perform certain operational tasks skills, the inherent properties of the weaponry system, decided by the quality characteristics of the system, the number of operational application methods.

The weaponry system different from the weaponry system, the decision the overall emergent of the system and non-linear of the traditional weaponry system operational capability polymerization general capability polymerization general method can not be applied to the combat capability polymerization, specific performance is as follows:

Weaponry system with a single weapon system does not have the overall emergent property, which means that the combat capability than the weapons and equipment, the system operational capability of the simple sum, but considering the relationship and interaction between the system and the system is formed the overall combat capability. Such as intelligence and reconnaissance capabilities firepower capability to have an impact on the role of the interaction between the protection capability and firepower interaction ${ }^{[4]}$.

Nonlinear relationship between the elements of the combat capability of the status and role of the various elements of the combat capability of non-equilibrium and interactions and constraints between them is not a simple relationship between addition and multiplication, but there is a positive feedback multiplier effect relationship. Such the combat capability of the large number of poor-quality equipment there is negative feedback saturation effects, and thus overall combat capability but weaker than a small amount of high-quality equipment ${ }^{[5]}$.

\section{B. Capability Polymerization Research}

Combat capability polymerization of weaponry and equipment system, mostly used component index system, through the level of the index system, reflect the overall combat capability and combat capabilities of each system, the relationship between the upper operational capability and lower operational capability, generally use the weighted sum or weighted product model. Traditional combat capability polymerization methods ignore the interaction between the system and cascade effect system, can not reflect the overall nonlinear emerged and combat capability elements, there is a certain one-sidedness ${ }^{[6]}$. 
Related areas of research scholars and experts on the operational capability of the weapon equipment system has been extensively studied, but most of them study for a weaponry system, Such as the study of the architecture of the weaponry the weaponry system evolution mechanism and overall emergence of the nature of the study was small.

The author introduced the concept of the feedback to system operational capability polymerization, the feedback mechanism between capacity indicators, is committed to resolve the combat capability and combat capability factor nonlinear polymeric system as a whole does not reflect the emergence of, which proposes a The weaponry system operational capability polymerization the new method.

\section{FEEDBACK MECHANISM}

\section{A. Feedback mechanism}

Feedback from the original to the basic concept of the concept of the control theory, refers to the output of the system is returned to the input terminal, and in some way to change the input thereby affecting system functionality.

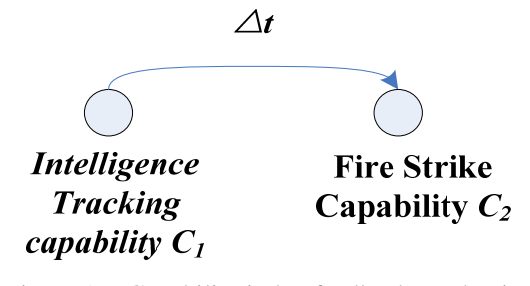

Figure 1. Capability index feedback mechanism

Intelligence investigation and firepower, for example, the ability of intelligence-driven investigations firepower capabilities have an impact, as shown in Figure1. Therefore, based on the feedback mechanism between capacity indicators, firepower capability can be expressed as

$$
C_{2}^{\prime}=C_{2}+\Delta t
$$

$\Delta t=f\left(C_{1}\right)$ amount of feedback, characterization $C_{1}$ capability $C_{2}$ capability level.

\section{B. Threshold-based feedback mechanism}

Cybernetics feedback principle, there is a positive feedback and negative feedback, positive feedback input has enhanced the input attenuation. Still intelligence detection capability and firepower capabilities, for example superior intelligence investigation has a great role in promoting the ability of precision fire strike capability, Event the intelligence investigation ability to meet the requirements, not only the firepower capacity will not help, it will not help, it will affect the play of the firepower capability. Therefore, in the feedback mechanism to introduce the concept of the threshold value, and when the input exceeds the threshold, the indicators will be associated with the ability of the multiplier effect of the positive feedback, and when the input is below the threshold value, will be associated with the ability index has weakening effect of negative feedback.
Based on the formula(1), the threshold-based feedback mechanism firepower capacity expressed as:

$$
C_{2}^{\prime}=\left\{\begin{array}{l}
C_{2}+\Delta t, C_{1} \geq \sigma \\
C_{2}-\Delta t, C_{1}<\sigma
\end{array}\right.
$$

$\Delta t=f\left(\left|C_{1}-\sigma\right|\right)$

capabilities $C_{1}$ degree of ability $C_{2}$ impact.

C. The feedback mechanism between multiple ability index

In the weaponry system capacity index system capacity indicators intricate relationships, a capacity indicators may be associated with multiple indicators, indicators of capacity in the feedback effects in other capacity indicators also accepted from other capacity indicators feedback. From simple to complex, ability index between the feedback can be divided into the following 3 .

- Unidirectional feedback. There exists only the ability index of competence indicators influence (see Figure 1 ).

- Feedback loop. The capacity index and capability index of mutual influence, interaction, as shown in figure 2. protection ability and firepower striking capability between interdependent, good protection ability to fire strike capability of the play, and good fire strike capability may contribute to the protection and survival.

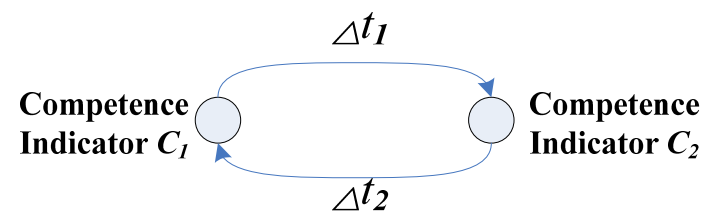

Figure 2. Feedback loop

- Complex feedback network. A plurality of capability index exists among a plurality of unidirectional feedback and a feedback loop, a plurality of capability index between them and the feedback arc is connected into a complex feedback network, as shown in figure 3 .

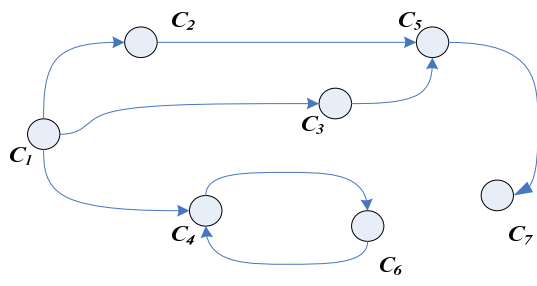

Figure 3. Complex feedback network

Feedback loop and complex feedback networks due to the existence of from input to output, and then from the output to the input of a closed loop, which is a constant 
feedback, gradually stabilizing process; Feedback loop and complex feedback networks relies on repeated iterative calculation, in order to achieve after reaching steady state capability index.

\section{POLYMERIZATION METHOD}

\section{A. Overall framework}

The emergence of non-linear characteristics of the elements of sexual and combat capability for weaponry system as a whole, I introduced the concept of cybernetics feedback system operational capability polymerization is proposed based on the feedback mechanism of the polymerization method, the general idea:

- From the weaponry system capacity needs departure construct hierarchical index system;

- According to the combat mission of the weaponry system, operational effectiveness and combat use of the way, in the same level indicator system to establish the relationship between the feedback network;

- Get the underlying indicators and quantized normalized processing;

- Indicators and the feedback network of every level of ability to iterate until the layer capacity index value stabilized;

- Combat capability polymeric layer, and ultimately the overall combat capability of the weapon equipment system. Figure 4 shows the ability to multi-level index system formed complex feedback network.

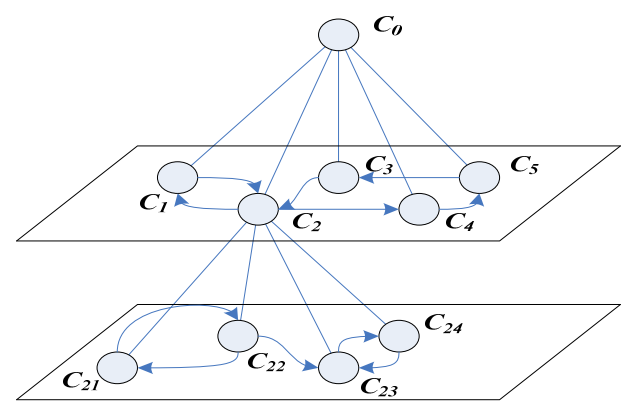

Figure 4. Polymerization method of the feedback

\section{B. The main processes and algorithms}

Process of polymerization methods based on the feedback mechanism is based on the index system assessment methods based on the introduction of a feedback mechanism between the indicators derived new system operational capability polymerization method, the method described below.

- Building index system

Build a scientific and reasonable index system is the prerequisite for the implementation of the system operational capability polymerization. Are many ways to build index system, common task-based analysis, based on capacity needs and based on the military value. The focus in work of this part of the contents of this article, from the needs of the operational capability of the weapon equipment system, the decomposed layer by layer, in order to establish the level of index system of combat capability.

- Relationship between the analysis of indicators, the establishment of the feedback network

The relationship between traditional indicators mean "or" relationship "and": the former description weighted model means lower operational capability indicators aggregated into upper combat capability indicators in cooperation with different weights; The latter describes the weighted models, for the upper combat capability, of each underlying operational capability indicators, although different weights, but they are indispensable, as long as a lower combat capability index is zero, and will result in the upper operational capability indicators zero.

The author feedback mechanism introduced in the same level of capability indicators constructed between the indicators and the indicator feedback arc, thereby forming the complex feedback network, as shown in Figure 4. The key to create a feedback network is: (1) The relationship between the analysis capability index build a feedback arc, from the point of view of the operational requirements or proceed from the interdependent relationship between the capacity indicators; (2) The determination of the feedback threshold, the feedback threshold is directly related to the critical point, the positive and negative feedback capability index value the merits of the boundary values can be obtained from the conventional data analysis refining; (3) The determination of the amount of feedback, the amount of feedback directly reflect the degree of association between competence indicators, to some extent reflects the multiplier effect of the weaponry architecture effect and force of combat capability, given by the experience of experts here.

- Underlying indicators quantify index weights calculated.

The underlying index is a description of the weaponry system capacity attributes. Quantification of the underlying indicators including the underlying indicators of access and normalization process. Description of the various capacity attributes mostly qualitative, it is not easy to give a specific, rigorous quantitative criteria can be different according to the indicators that describe the object, respectively, using quantitative methods, quantitative and qualitative combining methods and expertise given by.

Weight of index weight calculation, usually using the analytic hierarchy process, upcoming complexity of the system of indicators simplify judgment matrix element as representative of the ordered hierarchical structure expert experience to determine with mathematical computing combined together to identify indicators.

- Iterative calculation of the feedback network, layer by layer aggregate combat capability

Complex network of feedback from the input to the output due to the presence of closed-loop feedback from the output to the input, therefore the actual output of the feedback network is a constantly changing process of 
iterative computation; When the amount of change of the entire network is less than a present minimum value, that the network stabilized, then the output is the output of the system. If the capacity of a level indicators constitute a feedback network, i-ability indicators expressed ${ }_{\text {as }} C_{i}(i=1,2, \cdots, n)$; The initial value of $C_{i 0}$ by lower capacity indicators polymerization; previous iteration calculation to get value $C_{\text {ilast }}$; preset minimum value of $\mathcal{E}$, the feedback network iterative algorithm is described as follows.

$$
\begin{aligned}
& \text { Step 1: } C_{\text {ilast }}=C_{i 0} ; \\
& \text { Step 2: FOR } i=1,2, \cdots, n ; \\
& C_{i}=C_{i 0}+\Delta t_{i}, \quad \Delta t_{i}=\sum f\left(C_{\text {klast }}\right) \\
& k=1,2, \cdots, i-1, i+1, \cdots, n ; \\
& \text { Step 3: IF } \sum\left(C_{i}-C_{\text {ilast }}\right) \geq \varepsilon, i=1,2, \cdots, n, \\
& \text { THEN } C_{\text {ilast }}=C_{i} \text { GOTO Step 2; }
\end{aligned}
$$$$
\text { Step 4: OUTPUT } C_{i}, \quad i=1,2, \cdots, n \text { 。 }
$$

Based on the combat capability of the feedback mechanism polymerization method is layer polymerization process, the steps are as follows:

- First obtain the underlying index value, and the quantized normalization processing, the initial value of the underlying ability index.

- According to the relationship between the indicators for feedback iterative calculation to obtain the final value of the layer capability index;

- According to the relationship between the indicators for feedback iterative calculation to obtain the final value of the layer capability index;

Repeat (2) until the value of the overall combat capability of the system, the output of the final results.

With the proposed polymerization method based on a feedback mechanism, setting iteration preset minimum value of 0.001 , the feedback threshold of 5.0, the amount of feedback is $10 \%$. Results data as shown in TABLE1.

\section{TABLE I. DATA COMPARISON}

\begin{tabular}{|c|c|c|c|c|}
\hline Method & ICCA & DEWDC & IDAA & Total \\
\hline Traditional & 8.472 & 8.336 & 8.350 & 8.486 \\
\hline This method & 8.841 & 9.183 & 8.687 & 8.990 \\
\hline
\end{tabular}

After certification and repeatedly simulated combat drills in the demand for weapons and equipment, the combat capability of the reconnaissance and intelligence equipment expert assessment of 9.0, while the use of traditional combat capability polymerization method, ignoring the interaction between capacity indicators, the lack of non-line of combat capability consideration of sexual elements, the results of the assessment to 848.6. The polymerization method assessment results of the author proposes to 8.990 , more appropriate to the actual

\section{CONCLUSION}

The concept of feedback operational capability polymerization of the feedback mechanism between capacity indicators, the aggregation of the operational capability of the weapon equipment system based feedback mechanism. Example analysis results show that: the author proposed method can basically reflect the interaction between the various components within the system and cascade effect, reflecting the non-linear characteristics of the system as a whole to emerge and combat capability elements has a certain degree of effectiveness. The next step will build complex feedback network to study the ability to cross-level indicators, to further improve the operational capability of the weapon equipment system polymerization method.

\section{REFERENCES}

[1] X.F.Hu, J.Y.Yang, G.Y.Si. The war complex system simulation analysis and experimental. Beijing: National Defense University Press, 2008: 69-71.

[2] X.G.Niu National denfse system analysis of weaponry equipments. Beijing: National Defense Industry Press, 2007: 32.

[3] P.C.Luo,P.F.Fu, J.L.Zhou. Framework to evaluate the combat capability of weapons SoS. Systems Engineering and Electronics, 2005, 27(1): 72-75.

[4] G.Z.Bu. Study on the architecture and effectiveness for armament systems. Systems Engineering and Electronics, 2006, 28(10): 15441548.

[5] Hui.Z, P.C.Luo. Connectivity Evaluation for Weapon SOS Based on Incidence Matrix. Electrons Options \& Control, 2009, 16(2): 88-90.

[6] J.H.Zhang, F.Li, P.Di,h.Sun. Research on combat capability model of naval weaponry system. Journal of WUT(Information \& management engineering), 2009, 31(2): 314-317. 